\title{
Lumican as a novel potential clinical indicator for acute aortic dissection: A comparative study, based on multi-slice computed tomography angiography
}

\author{
GUORONG GU ${ }^{1 *}$, FANG WAN $^{2,3^{*}}$, YUAN XUE $^{1 *}$, WEIZHONG CHENG ${ }^{2}$, HAIYIN ZHENG $^{4}$, \\ YUN ZHAO $^{5}$, FAN FAN $^{1}$, YI HAN ${ }^{1}$, CHAOYANG TONG $^{1}$ and CHENLING YAO ${ }^{1}$
}

${ }^{1}$ Department of Emergency, Zhongshan Hospital, Fudan University; ${ }^{2}$ Shanghai Medical Imaging Institute, Shanghai 200032;

${ }^{3}$ Department of Sports Medicine and Arthroscopy Surgery, Huashan Hospital, Fudan University, Shanghai 200040;

Departments of ${ }^{4}$ Pathology, and ${ }^{5}$ Cardiac Surgery, Zhongshan Hospital, Fudan University, Shanghai 200032, P.R. China

Received October 9, 2014; Accepted September 18, 2015

DOI: $10.3892 /$ etm.2016.3020

\begin{abstract}
The aim of the present study was to investigate the association between serum lumican levels and acute aortic dissection (AAD) severity. A total of 82 patients with chest or back pain and 30 healthy volunteers were recruited. Among the patients, there were 70 cases of AAD and 12 cases of intramural hematoma (IMH). AAD severity was determined using multi-slice computed tomography angiography (MSCTA). Serum was collected from the patients upon admission, and lumican levels were detected using an enzyme-linked immunosorbent assay. In addition, correlation analyses were conducted between lumican levels and AAD severity by designing a 'SCORE X, RANGE Y' system to measure the number of affected vital arteries and vertical range of false lumen, based on the MSCTA. Lumican levels differed significantly among the AAD patients $(2.32 \pm 4.29 \mathrm{ng} / \mathrm{ml}), \mathrm{IMH}$ patients $(0.72 \pm 0.32 \mathrm{ng} / \mathrm{ml})$ and healthy volunteers $(0.85 \pm 0.53 \mathrm{ng} / \mathrm{ml} ; \mathrm{P}=0.003)$. In the AAD patients presenting within $12-72 \mathrm{~h}$ of symptom onset, the Spearman's rho correlation coefficient between lumican and SCORE or RANGE was $0.373(\mathrm{P}=0.046)$ and $0.468(\mathrm{P}=0.010)$, respectively. The present results suggest that lumican may be a potential marker for aiding the diagnosis and screening for AAD, and may be used to predict the severity of AAD.
\end{abstract}

\section{Introduction}

Acute aortic dissection (AAD) is a typical characteristic of acute aortic syndrome, which was once a potentially high-lethal

Correspondence to: Dr Chenling Yao, Department of Emergency, Zhongshan Hospital, Fudan University, 180 Fenglin Road Shanghai 200032, P.R. China

E-mail: guguorong1009@163.com

*Contributed equally

Key words: lumican, acute aortic dissection, multi-slice computed tomography angiography clinical situation (1-3). Currently, AAD is detectable within the early stages via minimally invasive, cross-sectional imaging techniques, including multi-slice computed tomography angiography (MSCTA) (4,5), magnetic resonance angiography and transesophageal echocardiography (6). In particular, the sensitivity of MSCTA for the diagnosis of aortic dissection has been reported to range between 83 and $100 \%$, and the specificity is reported to be $100 \%(4,7)$. With the prevalent utilization of MSCTA and advancements in image analysis software, patients with suspicious symptoms may be rapidly examined to confirm or eliminate the diagnosis of AAD. Notably, MSCTA may be used to evaluate the original location and range of the intimal tearing and the size of the false lumen and branch-vessel involvement, which are all associated with the patient's management and prognosis. Furthermore, AAD is currently a treatable disease due to recent advances in medical and surgical therapeutic approaches, such as endovascular aortic repair $(7,8)$.

The variability in disease presentation may obscure the diagnosis in certain cases $(2,3)$, and imaging modalities such as MSCTA remain prohibitive due to cost and availability. Therefore, further research is required to develop a rapid, inexpensive and accurate screening method for AAD. Limited progress in the biochemical diagnosis of AAD has been made in the last decade (9-11). Increases in a number of acute-phase proteins and coagulation parameters have been identified in AAD patients. However, the majority of these biomarkers are not specific to AAD, as they may be aberrantly expressed in other diseases $(9,10)$.

Lumican is a member of the small leucine-rich proteoglycan family that regulates the assembly and diameter of collagen fibers in the extracellular matrix of various tissues (12). Lumican expression correlates with a number of pathological conditions, including skin fragility, corneal opacification, and corneal and cardiac wound healing (13). Lumican is overexpressed in various tumor cell types (14), and its expression correlates with the growth and metastasis of various malignancies (15). To the best of our knowledge, our team demonstrated for the first time that lumican correlates with AAD (11). 
In the present study, serum lumican levels were compared with MSCTA manifestations to assess the association between those two factors in AAD patients. In addition, a semi-quantitative analysis was employed to evaluate the severity of AAD in patients, based on MSCTA results.

\section{Patients and methods}

Patient population. Patients with a sharp and unbearable recurrent/constant chest and/or back pain with a duration of at least 5 min each time were recruited. A total of 70 patients with AAD and 12 patients with aortic intramural hematoma (IMH) were selected in a consecutive manner at the Zhongshan Hospital Fudan University (Shanghai, China), and were matched with 30 healthy volunteers. Patients with trauma, syphilis, a creatinine level of $>355 \mu \mathrm{mol} / 1$, hemoglobin level of $<60 \mathrm{~g} / \mathrm{l}$ or systolic blood pressure of $<90 \mathrm{mmHg}$ prior to treatment were excluded. The diagnosis of AAD or IMH was confirmed using MSCTA. In cases with AAD, an intimal flap showing a tear of the intimal layer was observed. Unlike the false lumen in AAD cases, the crescent-shaped area observed in IMH patients was not enhanced subsequent to contrast agent administration, and no intimal tear was observed on contrast-enhanced CT scans (16). All study subjects provided their informed consent for participation in the study. The study was approved by the Ethics Committee of Zhongshan Hospital Fudan University. For each study subject, whole blood samples were immediately collected in BD Vacutainer SST tubes (BD Diagnostics, Plymouth, UK) following admission and were centrifuged at $2,770 \mathrm{x}$ g for $10 \mathrm{~min}$ at room temperature. The serum was frozen and stored in aliquots at $-80^{\circ} \mathrm{C}$ until required for analysis.

The Stanford system was used to describe the aortic dissection in the patients (17). If the dissection involved the ascending aorta and/or aortic arch, then patients were categorized as Stanford Type A. The type A patients generally require surgical treatment, such as the Bentall procedure which is performed to prevent the aorta from rupturing and causing a fatal hemorrhage. This surgical procedure involves replacing certain defective parts of the aorta, such as the valve or the upper part (also known as the ascending aorta), with a graft.

Enzyme-linked immunosorbent assay (ELISA) for measurement of lumican in serum and aortic sections. Serum levels of lumican were measured using ELISA kits from Cusabio Biotech Co., Ltd. (Wuhan, China; cat. no. CSB-E09797h). Briefly, each sample $(100 \mu \mathrm{l})$ was added to a 96-well microplate and incubated for $2 \mathrm{~h}$ at $37^{\circ} \mathrm{C}$. The liquid was then removed, and the biotin-antibody working solution $(100 \mu \mathrm{l})$ was added to each well and incubated for $1 \mathrm{~h}$ at $37^{\circ} \mathrm{C}$, after which the liquid was aspirated, and the wells were washed with $200 \mu$ l wash buffer three times. Horseradish peroxidase-avidin working solution $(100 \mu \mathrm{l})$ was added to each well and incubated for $1 \mathrm{~h}$ at $37^{\circ} \mathrm{C}$. Subsequent to five washes, $90 \mu 1$ 3,3',5,5'-tetramethylbenzidine was added to each well and incubated for $30 \mathrm{~min}$ at $37^{\circ} \mathrm{C}$. The enzymatic reaction was terminated by adding the stop solution. Thereafter, the absorbance was measured at $450 \mathrm{~nm}$ using a Bio-Rad model 680 microplate reader (Bio-Rad Laboratories, Inc., Hercules, CA, USA).
Aortic sections. To confirm the localization of the lumican protein in the human aortic sections, immunohistochemical staining and western blot analysis were performed. The aortic sections were obtained from 7 patients that had previously undergone the Bentall procedure and from 2 heart transplant donors, after obtaining patient consent.

Immunohistochemical staining. Immunohistochemical assay was performed according to the protocol of the Vectastain ABC kit (Vector Laboratories, Burlingame, CA, USA). Briefly, aortic tissue samples were fixed with freshly-prepared $4 \%$ paraformaldehyde and then dehydrated. The tissues were then embedded in molten paraffin and stored at room temperature until ready for sectioning. A microtome (RM2235; Leica Biosystems, Wetzlar, Germany) was used to cut the embedded tissue into 5-20- $\mu \mathrm{m}$ sections, which were then floated in a $50^{\circ} \mathrm{C}$ water bath. Next, the sections were mounted onto gelatin coated slides, allowed dry overnight and stored at room temperature until ready for staining. The sections were then deparaffinized, rehydrated and washed twice for $10 \mathrm{~min}$ with $1 \%$ animal serum in phosphate-buffered saline (PBS) with $0.4 \%$ Triton X-100 (PBS-T). Any non-specific binding was blocked by incubating the tissue sections with $5 \%$ serum in PBS-T for $30 \mathrm{~min}$ at room temperature, followed by incubation with polyclonal rabbit anti-human lumican antibody (Novus Biologicals, Littleton, CO, USA; cat. no. NBP1-87726; dilution, 1:200). Subsequently, the sections were incubated with ABC-HRP reagent (part of the Vectastain ABC kit; formed by mixing avidin with biotinylated horseradish peroxidase) for $1 \mathrm{~h}$ at room temperature and then washed twice in PBS for $10 \mathrm{~min}$ each time. The tissue sections were then counterstained with hematoxylin for $2 \mathrm{~min}$ and dehydrated, and mounting media (neutral balsam; Sangon Biotech Co. Ltd., Shanghai, China) was added.

Western blot analysis. For western blot analysis, the acute aortic dissection samples were lysed with lysis buffer (Sigma-Aldrich, St. Louis, MO, USA), total protein was extracted from the tissue samples, and the protein concentrations were determined using a protein assay kit (Bio-Rad Laboratories, Inc.). Next, $30 \mu \mathrm{g}$ protein was subjected to $10 \%$ SDS-PAGE and transferred to nitrocellulose membranes (Bio-Rad Laboratories, Inc.). The membranes were blocked with $5 \%$ non-fat milk for $1.5 \mathrm{~h}$. Subsequently, the samples were incubated with polyclonal rabbit anti-human lumican antibody (Abcam, Cambridge, UK; $1 \mu \mathrm{g} / \mathrm{ml}$; cat. no. ab98067) for the detection of lumican, followed by horseradish peroxidase-conjugated secondary antibodies (Thermo Fisher Scientific, Inc., Waltham, MA, USA; cat. no. A16104SAMPLE; dilution, 1:1,000). After incubation with secondary antibody, the proteins were detected with enhanced chemiluminescence reagents (Pierce Biotechnology, Rockford, IL, USA).

MSCTA data acquisition and imaging analysis. All patients and healthy volunteers underwent MSCTA imaging. Whole blood samples were collected immediately after admission; however, due to practical difficulties, MSCTA imaging was performed at a later time ( $<3 \mathrm{~h}$ after admission). All CT examinations were performed using a Somatom Definition AS MSCT system (Siemens Medical Solutions, Forchheim, Germany) 
Table I. Clinical features of all subjects.

\begin{tabular}{|c|c|c|c|c|}
\hline Clinical feature & AAD patients & IMH patients & Healthy volunteers & P-value \\
\hline Patients, $\mathrm{n}$ & 70 & 12 & 30 & - \\
\hline Age, years & $53.99 \pm 15.37$ & $59.17 \pm 8.17$ & $59.50 \pm 12.65$ & $0.148^{\mathrm{a}}$ \\
\hline Male gender, n (\%) & $49(70.00)$ & $8(66.67)$ & $16(53.33)$ & $0.275^{\mathrm{b}}$ \\
\hline Hypertension, n (\%) & $36(51.42)$ & $10(83.33)$ & $19(63.33)$ & $0.620^{\mathrm{b}}$ \\
\hline Marfan syndrome, n (\%) & $7(10.00)$ & $0(0.00)$ & $0(0.00)$ & $0.252^{\mathrm{b}}$ \\
\hline Time from onset to admission, $\mathrm{h}$ & $55.55 \pm 128.25$ & $41.08 \pm 65.03$ & - & $0.444^{\mathrm{c}}$ \\
\hline Systolic blood pressure, $\mathrm{mmHg}$ & $150.44 \pm 28.88^{\mathrm{d}}$ & $163.50 \pm 34.96^{\mathrm{d}}$ & $133.50 \pm 19.42$ & $0.002^{\mathrm{a}}$ \\
\hline Diastolic blood pressure, $\mathrm{mmHg}$ & $75.93 \pm 19.35$ & $86.17 \pm 10.71$ & $77.07 \pm 13.34$ & $0.167^{\mathrm{a}}$ \\
\hline In-hospital mortality, n (\%) & $8(11.42)$ & $0(0.00)$ & - & $0.218^{\mathrm{b}}$ \\
\hline
\end{tabular}

${ }^{\mathrm{a}}$ One-way analysis of variance; ${ }^{\mathrm{b}} \chi^{2}$ test; ${ }^{\mathrm{C}}$ Mann-Whitney test. ${ }^{\mathrm{d}} \mathrm{P}<0.05$ vs. healthy volunteers. AAD, acute aortic dissection; IMH, intramural hematoma.

using a standard clinical protocol. Scanning parameters were as follows: Voltage, $140 \mathrm{kV}$; effective mAs value per rotation, $400 \mathrm{mAs}$; gantry rotation time, 0.33-msec; collimation with z-flying focal spot for each detector, $128 \times 0.6 \mathrm{~mm}$. The pitch varied between 0.2 and 0.43 . For dose reduction, an automatic tube current modulation algorithm was used. A standard volume of $85 \mathrm{ml}$ contrast material (Isovue 370; Bracco Diagnostics, Inc., Seattle, WA, USA) was administered at $3 \mathrm{ml} / \mathrm{sec}$ followed by a $50-\mathrm{ml}$ saline flush. The automatic detection of peak enhancement in the descending aorta with a detection threshold of 120 Hounsfield units was used, while the helical scan was initiated automatically after the bolus detection. CT data sets were reconstructed at a slice thickness of $1 \mathrm{~mm}$.

Reconstructed images were transferred to a remote workstation and a cardiac function analysis software package (GE/Siemens Workstation: ADW 4.2, General Electric, Schenectady, NY, USA; Syngo MMWP VE36A, Siemens Healthcare, Erlangen, Germany) was used. The CT data sets were analyzed in a consensus reading by two experienced cardiovascular radiologists.

Data evaluation methods. Due to the complexity and inaccuracy of direct measurements, the vertical range of the false lumen was measured and we determined whether vital vessels were affected to estimate its surface area and the severity of AAD. Thus, a 'SCORE X, RANGE Y' system was designed to measure the number of groups of affected vital arteries and the vertical range of the false lumen. This system was designed by our group, based on the fact that the extent of the dissection and branch-vessel involvement may increase the morbidity and mortality (18).

SCORE grading depended on the number of affected vital vessels. The different groups of affected vital vessels were as follows: Group 1, including the brachiocephalic trunk, left common carotid artery and left subclavian artery; group 2, including the celiac trunk and superior mesenteric artery; and group 3, including the bilateral renal artery and bilateral iliac artery. If the affected vessel fell within one of the groups, the SCORE was marked as ' 1 '. If the affected vessel fell within two of the groups, the SCORE was marked as ' 2 '. If no vital vessels were affected, the SCORE was marked as ' 0 '. The maximal and minimal SCORE were 3 and 0 , respectively.

RANGE grading depended on the number of aortic segments that were affected by the false lumen. The aorta was divided into the following segments: i) Ascending to arch; ii) arch to celiac trunk level; iii) celiac trunk level to the level of the renal arteries; and iv) level of the renal arteries to the iliac arteries. If one segment was affected, RANGE was marked as ' 1 '. If two segments were affected, RANGE was marked as ' 2 ', If three segments were affected, RANGE was marked as ' 3 '. If all segments were affected, RANGE was marked as ' 4 '.

Statistical analysis. Data were collected and inputted in Excel (Microsoft Corporation, Redmond, WA, USA). All statistical analyses were performed using SPSS software, version 17.0 (SPSS, Inc., Chicago, IL, USA) and GraphPad Prism 5 software (GraphPad Software, Inc., San Diego, CA, USA), and the results were presented as mean \pm standard deviation. A comparative analysis of multiple groups was performed with a one-way analysis of variance or Mann-Whitney/Kruskal-Wallis test. Spearman's correlation coefficients were used to quantify the correlations. Bivariate correlation analyses were used to present the results, and $\mathrm{P}<0.05$ was considered to indicate a statistically significant difference.

\section{Results}

Clinical features. In total, 70 patients with AAD, 12 patients with IMH and 30 healthy volunteers were enrolled in the present study. The clinical features of all subjects are summarized in Table I. No statistically significant differences in age distribution, gender composition and past medical history (hypertension and Marfan syndrome, which is a genetic disorder of connective tissue resulting in defects of the heart valves and aorta, and occasionally aortic dissection) were observed among the three groups $(\mathrm{P}=0.148,0.275,0.620$ and 0.252 , respectively). Furthermore, there was no differences in the time from onset to admission between the AAD and IMH groups $(\mathrm{P}=0.331)$. However, systolic blood pressure was increased in the AAD and IMH patients compared with the healthy volunteers $(\mathrm{P}=0.002)$. 

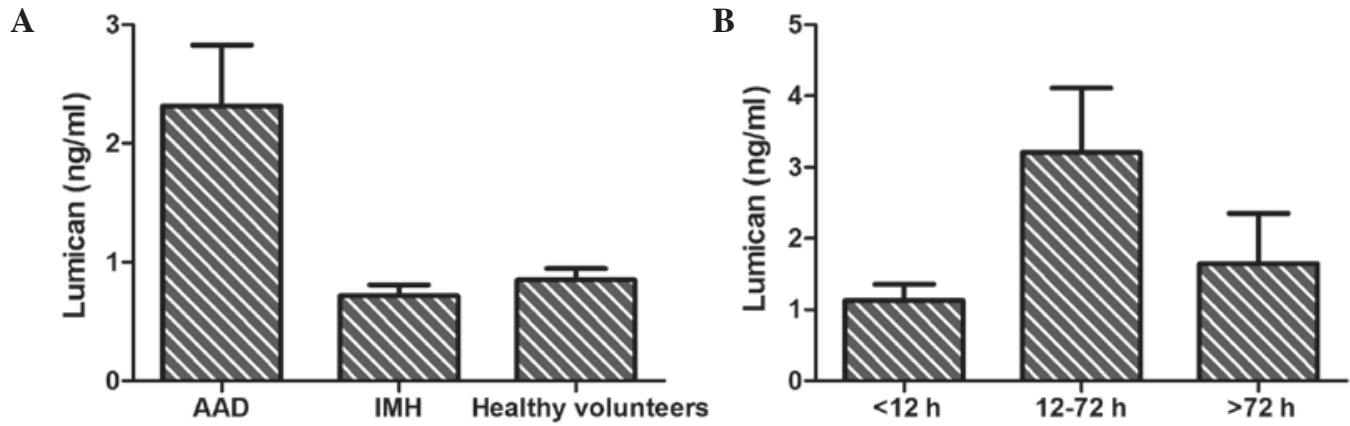

Figure 1. Serum levels of lumican analyzed among different groups. (A) Lumican levels differed significantly between the AAD patients, IMH patients and healthy volunteers $(\mathrm{P}=0.003$; data are presented as the mean \pm standard error). (B) Scatter diagram of time courses of lumican levels in the $70 \mathrm{AAD}$ patients. $\mathrm{AAD}$, acute aortic dissection; IMH, intramural hematoma.

A

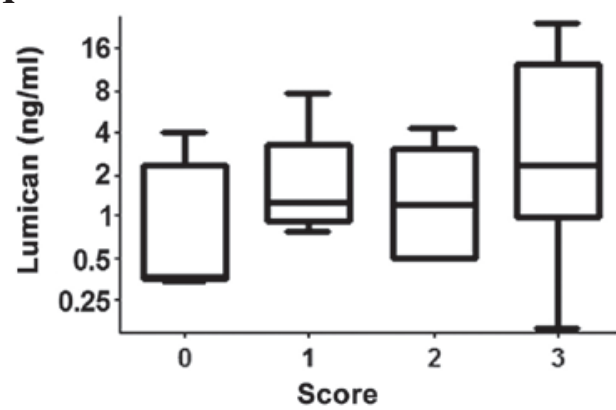

B

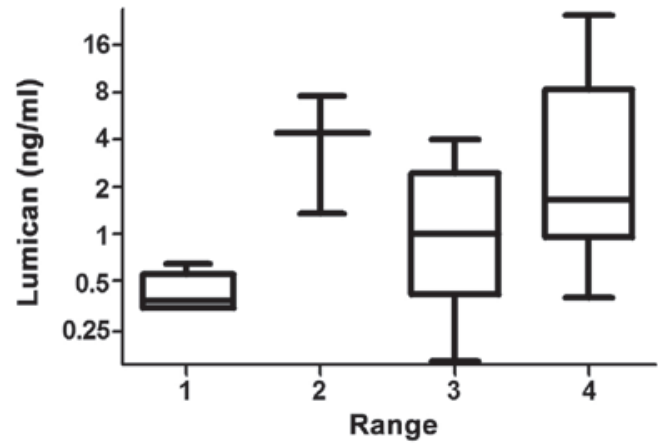

Figure 2. Box diagram of lumican levels in the serum of patients with acute aortic dissection (n=29). Serum lumican levels were correlated with the (A) SCORE $(\mathrm{r}=0.373, \mathrm{P}=0.046)$ or $(\mathrm{B}) \mathrm{RANGE}(\mathrm{r}=0.468, \mathrm{P}=0.010)$ values.

Serum levels of lumican. As shown in Fig. 1A, a statistically significant difference in serum lumican levels was detected among AAD patients $(2.32 \pm 4.29 \mathrm{ng} / \mathrm{ml})$, IMH patients $(0.72 \pm 0.32 \mathrm{ng} / \mathrm{ml})$ and healthy volunteers $(0.85 \pm 0.53 \mathrm{ng} / \mathrm{ml}$; $\mathrm{P}=0.003)$. Furthermore, the association between the level of serum lumican and the time from symptom onset (Fig. 1B) in AAD patients was assessed, and a significant difference was observed between patients presenting at the hospital within $12-72 \mathrm{~h}(3.21 \pm 5.57 \mathrm{ng} / \mathrm{ml})$ and those presenting within $<12 \mathrm{~h}$ $(1.13 \pm 1.10 \mathrm{ng} / \mathrm{ml})$ or $>72 \mathrm{~h}(1.65 \pm 1.99 \mathrm{ng} / \mathrm{ml} ; \mathrm{P}=0.005)$ from the symptom onset.

Correlation of lumican levels with SCORE and RANGE. In total, 8 patients presented at $>72 \mathrm{~h}$, including 4 patients with Stanford Type A disease, of which 1 patient underwent the Bentall procedure, and 4 patients with Stanford Type B disease, of which 1 patient received endovascular graft exclusion treatment. Among the 24 patients that presented at $<12 \mathrm{~h}, 7 / 14$ Type A and 7/10 Type B patients were treated surgically. As the serum lumican levels are significantly associated with the time from symptom onset, further analysis was only performed in 38 patients that presented within $12-72 \mathrm{~h}$ after an episode of chest/back pain (of these, 9/25 Type A and 8/13 Type B patients were treated surgically). In total, 9 subjects were excluded as their diagnosis was not confirmed by MSCTA or MSCTA data acquisition was obtained at $>3 \mathrm{~h}$ following admission. Among the remaining $29 \mathrm{AAD}$ patients, 7/16 Stanford Type A patients had undergone the Bentall procedure, and 8/13 Stanford Type B patients had received endovascular graft exclusion treatment.
As shown in Fig. 2A, the lumican levels in patients with SCORE values of $0,1,2$ and 3 were $1.15 \pm 1.61,2.31 \pm 2.61$, $1.64 \pm 1.57$ and $6.63 \pm 8.43 \mathrm{ng} / \mathrm{ml}$, respectively. The Spearman's rho correlation coefficient between lumican levels and SCORE was $0.373(\mathrm{P}=0.046)$, which indicated that the level of lumican was correlated with the number of affected vital vessels. In patients with RANGE values of 1,2, 3 and 4 (Fig. 2B), the serum lumican levels were $0.43 \pm 0.14,4.46 \pm 4.41$, $1.45 \pm 1.40$ and $5.57 \pm 7.58 \mathrm{ng} / \mathrm{ml}$, respectively. In addition, the correlation coefficient between lumican levels and RANGE was $0.468(\mathrm{P}=0.010)$, which indicated that lumican levels were correlated with the range of the false lumen.

Immunohistochemical staining and western blot analysis. Among the 29 eligible AAD patients presenting within 12-72 $\mathrm{h}$ after onset, 7 Standford Type A patients had received the Bentall procedure. Immunohistochemical staining and western blot analysis of lumican were performed in the aortic sections from the 7 patients that had previously undergone the Bentall procedure and from 2 heart transplant donors. Western blot analysis (Fig. 3A) and immunohistochemical staining (Fig. 3B and C) were performed to detect the expression of lumican in the aortic sections of AAD patients and donors.

Typical case. Images demonstrating the expression of lumican from the aortic sections of a 37-year-old male patient are shown in Fig. 3B. This patient had no history of hypertension or family history of aortic dissection, and presented with sharp 
A

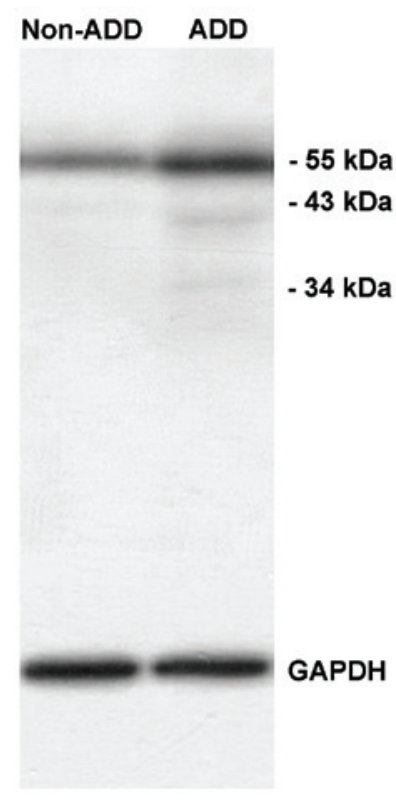

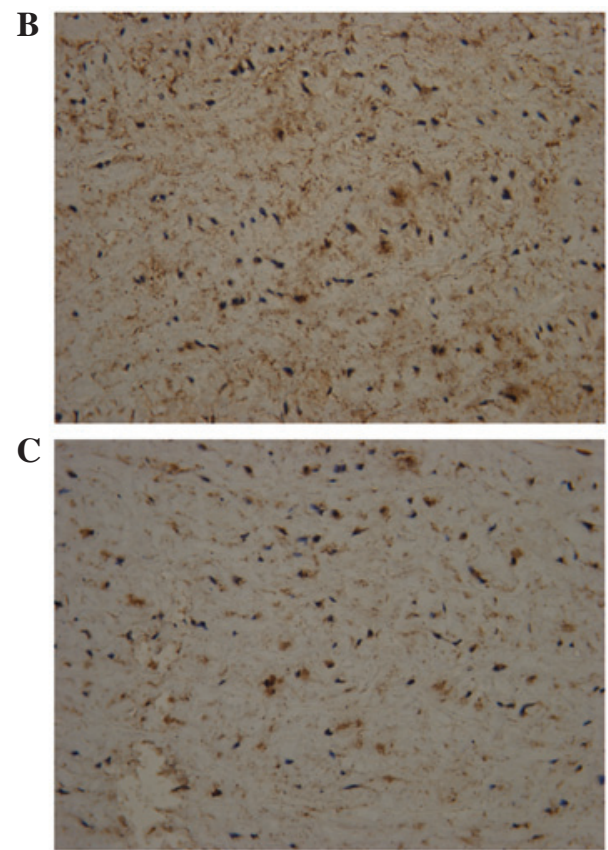

Figure 3. (A) Western blot analysis of lumican expression in a typical patient (lane AAD) and a male heart transplant donor (lane Non-AAD) aorta. The expression of lumican (brown) in the aortic medial layer of (B) the patient and (C) the donor was assayed by immunohistochemical staining (magnification, $\mathrm{x} 200)$. AAD, acute aortic dissection.

chest pain for $72 \mathrm{~h}$, following which he underwent a Bentall procedure and survived. The evaluation for this patient was SCORE 3 and RANGE 4, and the serum lumican level was $12.54 \mathrm{ng} / \mathrm{ml}$. Fig. 3B shows the expression of lumican (brown) in the aortic medial layer (magnification, x200). The expression of lumican in the aortic medial layer of a 24-year old male heart transplant donor is shown in Fig. 3C (magnification, x200). Although lumican was expressed in the aortic medial layer of AAD patients and healthy donors, the serum levels of lumican were elevated in AAD patients, but not in the healthy donors, which may reflect our hypothesis that the medial layer released more lumican into the circulation via the blood flow from the intimal tear.

\section{Discussion}

Although AAD is uncommon, it may rapidly evolve into a serious cardiovascular condition if not detected rapidly. Thus, a specific biomarker test or array that can be used as a diagnostic tool to reliably include or exclude AAD is required. As AAD affects the aortic medial layer, the search for biomarkers has focused on the markers that are associated with the vascular smooth muscle (myosin), vascular interstitium (calponin), elastic laminae (soluble elastin fragments) of the aorta, or secondary phenomena due to the exposure of blood to nonintimal vascular surfaces (D-dimers) (19). In 2004, a study demonstrated that extracellular matrix components of the vessel walls, such as elastin, may be elevated in aortic dissections (10); however, these are nonspecific biomarkers for AAD (9).

Lumican is distributed in interstitial collagenous matrices (ICMs) throughout the body. In coronary artery ischemic lesions, lumican is overexpressed by vascular smooth muscle cells (20) and is synthesized in aortic smooth muscle cells (21). The results of our previous proteomic study indicated that lumican may be a potentially specific marker for AAD (13). In the present study, the results demonstrated that the level of lumican in patients with AAD was significant higher compared with that in patients with IMH and healthy volunteers, and the levels were 2.32 \pm 4.29 , $0.72 \pm 0.32$ and $0.85 \pm 0.53 \mathrm{ng} / \mathrm{ml}$, respectively $(\mathrm{P}=0.003)$.

Furthermore, in the present study, the expression of lumican was detected in the aortic sections of healthy donors and AAD patients, and the serum level of lumican did not significantly differ between IMH patients without an intimal tear and healthy volunteers. These results suggest that an increased number of ICMs are exposed to the circulation in cases featuring an intimal tear, which may result in a false lumen. Due to the exposure of ICMs to the circulation, lumican is released into the circulation, thus allowing for it to be identified by laboratory tests.

Theoretically, the level of serum lumican is correlated with the surface area of exposed ICMs. Therefore, we hypothesize that the level of serum lumican may be used to predict the size and range of lesions, in addition to the severity of AAD in afflicted patients.

Currently, $>70 \%$ of AAD patients are diagnosed following evaluation using MSCTA, since these patients show typical signs of aortic dissection, including false lumen and the intima tear (16). Notably, these signs may additionally indicate other pathological entities, such as IMH. As the extent of the dissection and branch-vessel involvement may increase morbidity and mortality (18), the current study evaluated the extent of the dissection and the branch-vessel involvement. Lumican levels were elevated in AAD patients, but not in IMH patients, which may reflect the pathological process by which the blood enters through the intimal tear, thereby releasing lumican into the circulation. Furthermore, the level of lumican in the serum was correlated with the SCORE $(r=0.373, \mathrm{P}=0.046)$ or RANGE $(r=0.468, P=0.010)$ values, thus indicating that patients with 
higher levels of lumican exhibit increased disruption of the aortic media layer and more marked branch-vessel involvement.

As an initial step, the results of the current study indicated that lumican may be a potential novel serum biomarker for AAD. However, the ultimate development of biomarkers that provide sufficient sensitivity or specificity for the diagnosis of AAD may require multiple validations and clinical studies. The current study is merely a perspective study due to the limited number of samples, which is the primary limitation of the study. Due to the insufficient recruitment of patients, any association between lumican and the quantity of intra-false lumen thrombus formation cannot be confirmed, while the diagnostic value is also uncertain. An expanded patient population is required in order to confirm the present results and conduct further investigations. However, the present study provided preliminary indications of the diagnostic capacity of lumican, which require validation.

In conclusion, the present results demonstrated that serum lumican levels were higher in AAD patients compared with those in IMH patients or healthy volunteers. Therefore, lumican may be a useful marker for the diagnosis of and screening for AAD. In addition, the level of lumican in the serum may be a potential predictor of the severity of AAD, as it was associated with the lesion range, as confirmed by MSCTA.

\section{Acknowledgements}

This study was supported by a grant from the Shanghai Committee of Science and Technology (no. 114119A9000).

\section{References}

1. Mészáros I, Mórocz J, Szlávi J, Schmidt J, Tornóci L, Nagy L and Szép L: Epidemiology and clinicopathology of aortic dissection. Chest 117: 1271-1278, 2000.

2. Hagan PG, Nienaber CA, Isselbacher EM,Bruckman D, Karavite DJ, Russman PL, Evangelista A, Fattori R, Suzuki T, Oh JK, et al: The international registry of acute aortic dissection (IRAD): New insights into an old disease. JAMA 283: 897-903, 2000.

3. Elefteriades JA, Barrett PW and Kopf GS: Litigation in nontraumatic aortic diseases-a tempest in the malpractice maelstrom. Cardiology 109: 263-272, 2008

4. Hayter RG, Rhea JT, Small A, Tafazoli FS and Novelline RA: Suspected aortic dissection and other aortic disorders: Multi-detector row CT in 373 cases in the emergency setting. Radiology 238: 841-852, 2006.

5. Stein PD, Fowler SE, Goodman LR, Gottschalk A, Hales CA, Hull RD, Leeper KV Jr, Popovich J Jr, Quinn DA, Sos TA, et al: Multidetector computed tomography for acute pulmonary embolism. N Engl J Med 354: 2317-2327, 2006.
6. Macura KJ, Szarf G, Fishman EK and Bluemke DA: Role of computed tomography and magnetic resonance imaging in assessment of acute aortic syndromes. Semin Ultrasound CT MR 24: 232-254, 2003.

7. Iezzi R and Cotroneo AR: Endovascular repair of abdominal aortic aneurysms: CTA evaluation of contraindications. Abdom Imaging 31: 722-731, 2006.

8. Rousseau H, Chabbert V, Maracher MA, El Aassar O, Auriol J, Massabuau $\mathrm{P}$ and Moreno R: The importance of imaging assessment before endovascular repair of thoracic aorta. Eur J Vasc Endovasc Surg 38: 408-421, 2009.

9. Suzuki T, Distante A and Eagle K: Biomarker-assisted diagnosis of acute aortic dissection: How far we have come and what to expect. Curr Opin Cardiol 25: 541-545, 2010.

10. Shinohara T, Suzuki K, Okada M, Shigai M, Shimizu M, Maehara T and Ohsuzu F: Soluble elastin fragments in serum are elevated in acute aortic dissection. J Cardiol 43: 96-97, 2004 (In Japanese)

11. Gu G, Cheng W, Yao C, Yin J, Tong C, Rao A, Yen L, Ku M and Rao J: Quantitative proteomics analysis by isobaric tags for relative and absolute quantitation identified lumican as a potential marker for acute aortic dissection. J Biomed Biotechnol 2011: 920763, 2011

12. Takayama R, Ishiwata T, Ansai S, Yamamoto T, Matsuda Y, Naito $Z$ and Kawana S: Lumican as a novel marker for differential diagnosis of Bowen disease and actinic keratosis. Am J Dermatopathol 35: 827-832, 2013.

13. Engebretsen KV, Lunde IG, Strand ME, Waehre A, Sjaastad I, Marstein HS, Skrbic B, Dahl CP, Askevold ET, Christensen $\mathrm{G}$, et al: Lumican is increased in experimental and clinical heart failure and its production by cardiac fibroblasts is induced by mechanical and proinflammatory stimuli. FEBS J 280: 2382-2398, 2013

14. Yang ZX, Lu CY, Yang YL, Dou KF and Tao KS: Lumican expression in pancreatic ductal adenocarcinoma. Hepatogastroenterology 60: 349-353, 2013.

15. Nikitovic D, Papoutsidakis A, Karamanos NK, and Tzanakakis GN: Lumican affects tumor cell functions, tumor-ECM interactions, angiogenesis and inflammatory response. Matrix Biol 35: 206-214, 2014.

16. Erbel R, Alfonso F, Boileau C, Dirsch O, Eber B, Haverich A, Rakowski H, Struyven J, Radegran K, Sechtem U, et al: Diagnosis and management of aortic dissection. Eur Heart J 22: 1642-1681, 2001.

17. Sheikh AS, Ali K and Mazhar S: Acute aortic syndrome. Circulation 128: 1122-1127, 2013.

18. Castañer E, Andreu M, Gallardo X, Mata JM, Cabezuelo MA and Pallardó Y: CT in nontraumatic acute thoracic aortic disease: Typical and atypical features and complications. Radiographics 23: S93-S110, 2003.

19. Nazerian P, Morello F, Vanni S, Bono A, Castelli M, Forno D, Gigli C, Soardo F, Carbone F, Lupia E and Grifoni S: Combined use of aortic dissection detection risk score and D-dimer in the diagnostic workup of suspected acute aortic dissection. Int J Cardiol 175: 78-82, 2014.

20. Qin H, Ishiwata T and Asano G: Effects of the extracellular matrix on lumican expression in rat aortic smooth muscle cells in vitro. J Pathol 195: 604-608, 2011.

21. Naito Z: Role of small leucine-rich proteoglycan (SLRP) family in pathological lesions and cancer cell growth: J Nippon Med Sch 72: 137-145, 2005. 